\title{
Resiliencia en estudiantes de secundaria oaxaqueños
}

\section{Resilience in middle school oaxaqueños students}

David Israel Becerra Martín

Yanko Norberto Mézquita Hoyos

Universidad Autónoma de Yucatán
José Ignacio Cuitún Coronado

Colegio Libre de Estudios Universitarios

\section{Resumen}

El objetivo principal de este trabajo fue comparar la validación de la escala de resiliencia, utilizada por Villegas y Zamudio (2006), con un nuevo formato de respuestas y una nueva muestra de estudiantes. El instrumento mencionado fue una escala de 34 items con 10 rangos de respuesta en forma de porcentajes, con un Alfa de Cronbach de 0.83, administrado a 294 estudiantes (147 mujeres y 149 hombres) de una secundaria del estado de Yucatán. De esta manera, se obtuvieron los factores de Autoestima, Moralidad, Apoyo Social, Independencia, Relacionarse, Apoyo Familiar y Optimismo. Para fines de la comparación propuesta, se aplicó un diseño cuantitativo y transeccional a una muestra de 250 estudiantes del sistema de telesencundaria del stado de Oaxaca, (111 mujeres y 139 hombres). El instrumento utilizado fue la misma escala de resiliencia, de 34 ítems de Villegas y Zamudio (2006), pero se modificó el rango de respuestas hacia un formato tipo Likert con cinco rangos de respuestas que fueron: 1: Nada parecido a mí, 2: Poco parecido a mí, 3: Algo parecido a mí 4: Bastante parecido a mí, y 5: Muy parecido a mí. Al analizar los datos de la nueva muestra, se observó que la consistencia interna de la escala de resiliencia puntuó con un alfa de Cronbach de 0.87 y al realizar el análisis factorial se obtuvieron los factores: Autoestima, Moralidad, Apoyo Social, Autopercepción e Independencia. Con base a lo anterior, se concluyó que existe semejanza entre las dimensiones obtenidas en este estudio, con respecto a las que reportaron los autores mencionados.

Palabras clave: resiliencia, psicología positiva, factores protectores, adolescentes, telesecundaria.

Nota del autor

David Israel Becerra Martín, Facultad de Educación, Universidad Autónoma de Yucatán (UADY); Yanko Norberto Mézquita Hoyos, Facultad de Educación, UADY; José Ignacio Cuitún Coronado, Colegio Libre de Estudios Universitarios.

La correspondencia en relación a este artículo debe dirigirse a David Israel Becerra Martín, Facultad de Educación, UADY, carretera Mérida-Tizimin, Km 1, Cholul. Mérida, Yucatán, México.

Dirección electrónica: davidbecerramartin@gmail.com 


\begin{abstract}
The main objective of this work was to compare the validation of the resilience scale, used by Villegas and Zamudio (2006), with a new format of answers and a new sample of students. The instrument was a 34 item scale with a 10 response ranges in percentages, with a Cronbach's alpha of 0.83, applied to 294 students (147 women and 149 men) from a Yucatan state middle school and produced the factors Self-esteem, morality, social support, independence, relationship, family support and optimism. For the purposes of the comparison, a quantitative and transectional design was applied to a sample of 250 students from the state school system of Oaxaca (111 women and 139 men). The instrument used was the same Resilience scale, of 34 items of Villegas and Zamudio (2006), but modifying its range of responses to a Likert format with five ranges of responses that were: 1: Nothing like me, 2: Little like me, 3: Something like me 4: Quite a bit like me, 5: Very similar to me. When analyzing the data of the new sample, it was observed that the internal consistency of the Resilience scale scored with a Cronbach's alpha of 0.87 and when the factor analysis was performed, the following factors were obtained: self-esteem, morality, social support, self-perception and independence. It was concluded that there is similarity between the dimensions obtained in this study, with respect to those reported by Villegas and Zamudio, (2006).
\end{abstract}

Keywords: resilience, positive psychology, protective factors, teenages, telesecundaria.

De manera natural, la vida es un proceso que conlleva una serie de retos y dificultades a lo largo de sus diferentes etapas, sin embargo, algunas personas se enfrentan a factores de riesgo que incrementan las problemáticas y que impactan directamente en su salud física y mental. Es decir, el entorno contribuye al desarrollo de pensamientos, sentimientos $y$ conductas más negativas, nocivas y violentas, tanto para los individuos mismos como para los demás (Cassaretto \& Martínez, 2009).

En la psicología, tanto el enfoque psicoanalítico (como es citado en Ardila, 1971), como el conductual (Yates, 1982), se han enfocado en estudiar los efectos negativos que producen las situaciones adversas sobre las personas y la forma cómo éstos afrontan dichas situaciones; mientras tanto, dejan de lado los enfoques en los que el centro de la atención es el desarrollo de habilidades, la búsqueda de factores protectores y el estudio de fenómenos asociados con la salud más que la enfermedad (Carretero, 2010).

Sin embargo, a mediados del siglo $\mathrm{XX}$ se observó que existen personas que no se ven afectadas por situaciones adversas como violencia familiar y social, duelos, pérdidas o pobreza, por mencionar algunos; y más aún, salían avante y se integraban de forma activa a la sociedad, mientras que otros, en las mismas situaciones no lo lograban. Así se inició un cambio de paradigma en los estudios de la psicología y surgió la psicología positiva, cuya atención se enfoca ahora en características 
humanas positivas como la capacidad de sobreponerse al dolor, el humor, creatividad, optimismo, la felicidad, el bienestar y la satisfacción con la vida (Cassaretto \& Martínez, 2009; Seligman, 2003), y más recientemente se ha vinculado con un éxito moderado, la resiliencia con la tenacidad (Becerra, Espíndola, \& Mézquita, 2012).

En este sentido, con el surgimiento de la psicología positiva, se comenzó a tomar más en cuenta a la persona como un ser con potencial emocional, y se formalizaron varios estudios en los que el objeto de interés era la capacidad emocional que tienen las personas y que les permiten resistir y fortalecerse ante las situaciones adversas, además de construir un presente y un futuro positivo (Seligman, 2003; Vera, 2006). Estos trabajos fueron iniciados por investigadores como Rutter, quien se enfocó en la flexibilidad social para la adaptación (Naranjo, 2010).

Cyrulnik (2001, como se citó en Naranjo, 2010) estudió a los sobrevivientes de campos de concentración e identificó la capacidad psicológica, en algunas de estas personas, para sobreponerse a episodios de dolor emocional o grandes contratiempos; asimismo, definió la resiliencia psíquica y refirió que esta característica era el "resultado de múltiples procesos mentales que contrarrestan las situaciones nocivas" (p. 271). En dicho sentido, Navarrete (2009) señala que la resiliencia consiste en la capacidad que tienen las personas para salir exitosos a pesar de estar dentro de circunstancias adversas, así como para poder recuperarse después de pasar por situaciones estresantes y regresar a sus actividades cotidianas con éxito. Otras definiciones importantes son las referidas por Grotberg (2006), quien la define como la capacidad de enfrentar, sobreponerse y fortalecerse $\mathrm{y} / \mathrm{o}$ transformarse debido a experiencias de adversidad; en tanto Vanistendael (1996, citado en González-Arratia, Valdez, \& Zavala, 2008), como la cualidad que les permite a las personas tener éxito de modo aceptable para la sociedad a pesar de atravesar por situaciones de estrés o adversas, las cuales implican sufrir riesgos negativos.

De las definiciones consultadas, se puede observar que éstas coinciden en que la resiliencia implica enfrentarse a una situación adversa, y la consecuente capacidad de salir adelante, es decir, existe un proceso que ocurre entre una adversidad y una superación positiva de dicha situación (Kalawski \& Haz, 2003). Por lo tanto, dicho constructo se considera como un rasgo personal cultivado en la historia del individuo, o bien, es la forma cómo él se apropia de su realidad, lo cual le permite desarrollar comportamientos saludables, así como superar, dichas situaciones (Saavedra \& Villalta, 2008).

Dado lo anterior, al estudiar la resiliencia, de acuerdo a Vanistendael (1997, citado en Matalinares et al., 2011), hay que distinguir cinco dimensiones imprescindibles en el proceso para ser una persona resiliente, a saber:

1. Existencia de redes sociales informales: la persona tiene amigos, participa de 
actividades con ellos y lo hace con agrado; tiene, en general, una buena relación con los adultos.

2. Sentido de la vida, trascendencia: la persona muestra capacidad para descubrir un sentido y una coherencia en la vida.

3. Autoestima positiva: la persona se valora a sí misma, confía en sus capacidades y muestra iniciativa para emprender acciones o relaciones con otras personas porque se siente valiosa y merecedora de atención.

4. Presencia de aptitudes y destrezas: es capaz de desarrollar sus competencias y confiar en ellas.

5. Sentido del humor: la persona es capaz de jugar, reír y gozar de las emociones positivas, es capaz de disfrutar de sus experiencias.

Conforme a Vanistendael (1997, citado en Matalinares et al., 2011), las dimensiones citadas permiten conocer las características a considerar en el momento de estudiar o medir la resiliencia; a lo que cabe añadir, las dimensiones propuestas se encuentran dentro de un contexto de estudios de diversos instrumentos de medición, los cuales conllevan sus propias dimensiones, por ejemplo: Crespo, Fernández-Lansac y Soberón (2014) proponen las siguientes: Afrontamiento y persistencia ante situaciones de estrés, Capacidad de superación y Logro de objetivos, Valoración positiva y Confianza. González-Arratia, Valdez y González-Escobar (2014), refieren diversas escalas con sus respectivas dimensiones: como es el caso de Jew, Green \& Coger (1992), quienes postulan las dimensiones: Independencia-riesgo, Orientación futura y Optimismo; para Hurtes y Allen (2001), la resiliencia está integrada por los factores de: Creatividad, Relaciones e Iniciativa; para Friborg, Hjemdal, Rosenvinge y Martinussen (2001), la resiliencia la componen las dimensiones de: Competencia personal, Competencia social, Coherencia familiar, Apoyo social y Estructura personal. Por su parte González-Arratia y Valdez (2012), proponen los factores: Protectores Internos, Externos y de Empatía como los que más se han identificado con el perfil de la persona resiliente.

Por otra parte, Villegas y Zamudio (2006), con una escala proporcionada por Valdez y González (comunicación personal, enero, 2005), encontraron que las dimensiones de la resiliencia fueron: Autoestima, Moralidad, Apoyo social, Independencia, Capacidad para relacionarse, Apoyo familiar y Optimismo. Merece resaltarse que esta escala tuvo un formato de respuestas con base en porcentajes y 10 rangos obtenidos de los mismos. Sobresale que en el estudio mencionado se encontró un nivel de resiliencia de $67.39 \%$ y sólo se encontraron diferencias entre estudiantes del sexo masculino y femenino en los factores de Autoestima e Independencia a favor de los estudiantes del sexo masculino. Con base en la escala mencionada, para poder conocer más sobre este constructo, quienes esto escriben se propusieron: 
1. Modificar el formato de respuestas de la escala citada;

2. Determinar el nivel de resiliencia en una muestra de estudiantes de Oaxaca;

3. Comparar la estructura de respuestas de la muestra mencionada con la obtenida por Villegas y Zamudio (2006) y

4. Comparar la resiliencia entre estudiantes del sexo masculino y femenino.

\section{Método}

Esta investigación tiene un diseño cuantitativo y transeccional, ya que el objetivo del estudio es conocer el comportamiento de los factores de la variable Resiliencia, en una población con la que poco se ha trabajado este tipo de investigaciones, en un momento determinado en específico (Hernández, Fernández, \& Baptista, 2015).

\section{Participantes}

La escala fue aplicada a estudiantes de 2 do., y 3er., año del sistema de telesencundaria del estado de Oaxaca, en 14 comunidades de las regiones Mixteca, Zapoteca y Chatina. Una vez delimitada la población, se realizó un muestreo no probabilístico por conveniencia, siendo conformada por 250 alumnos, de los cuales 139 son hombres $(55.6 \%)$ y 111 , mujeres (44.4\%), con edades entre los 12 y 18 años, con una media de 14.74 años y una desviación estándar de 0.99 .

\section{Instrumentos}

Se utilizó la escala de Resiliencia de Valdez y González (2005, citada en Villegas \& Zamudio 2006), conformada por 34 afirmaciones con un formato de respuestas de porcentajes y con 10 rangos de las mismas. La escala mencionada estuvo integrada por los factores ya mencionados, a los cuales se añadió su respectivo alfa de Cronbach: Autoestima (.90), Moralidad (.77), Apoyo social (.69), Independencia (.72), Capacidad para relacionarse (.64), Apoyo familiar (.59) y Optimismo (.65), con un alfa global de .83. Para fines de este estudio, se modificó el rango de respuestas de la escala citada hacia un formato de tipo Likert, con cinco rangos de respuestas, a saber: 1: Nada parecido a mí, 2: Poco parecido a mí, 3: Algo parecido a mí 4: Bastante parecido a mí, y 5: Muy parecido a mí.

\section{Procedimiento}

Para la aplicación de esta escala se capacitó a los docentes de cada comunidad, con el fin de que realizaran la aplicación a sus propios alumnos, por salón. Más tarde se recolectaron los resultados para su análisis e interpretación.

\section{Resultados}

A continuación, se presentan los análisis de frecuencias, de contingencias, de discriminación, factorial y de consistencia interna del cuestionario implementado. $\mathrm{El}$ análisis de frecuencias de las opciones de respuesta por 
cada reactivo, evidenció que ningún reactivo mostró alguna opción elegida más de $80 \%$ de las veces y a su vez todos mostraron alguna opción con menos de un $20 \%$ de elecciones, las cuales generalmente fueron las opciones de menor valor para fines de este cuestionario: Nada parecido a mí y Poco parecido a mí.

De tal forma, se hizo una tabla de contingencias entre $25 \%$ superior y $25 \%$ inferior de la sumatoria del total del puntaje de la escala, contra las frecuencias de elección de cada opción, de esta operación una consistente tendencia: $25 \%$ superior de la sumatoria total de la escala se asoció con las opciones de valor más alto de cada reactivo para fines de este cuestionario: Bastante parecido a mí y Muy parecido a mí, en tanto $25 \%$ inferior se vinculó con los puntajes bajos de las opciones utilizadas: Nada parecido a mí y Poco parecido a mí.

Posteriormente, con ayuda de una prueba t-student se evaluó: si $25 \%$ superior y $25 \%$ inferior de la sumatoria del puntaje total de la escala, discriminaba entre las opciones elegidas de cada reactivo; se determinó que las opciones elegidas de todos los reactivos arrojaron una diferencia estadística significativamente entre los puntajes extremos de la escala.

El siguiente paso fue la realización del análisis factorial, a través del método de Factores Principales (ejes principales) con rotación varimax. Se extrajeron los factores que tuvieran valores propios mayores a uno, así como también se suprimieron los valores absolutos menores a .5. Una vez obtenidos los factores, se tomaron los valores rotados, sólo se consideraron aquellos factores conformados por tres o más reactivos. En el caso de aquellos factores que tuvieran reactivos en común, se decidió ubicar el reactivo en el factor donde tuviera congruencia con los demás reactivos, de lo anterior, se obtuvieron ocho factores, los cuales explican $59.46 \%$ de la varianza (tabla 1 ).

Una vez obtenidos los factores, se realizó un análisis de los reactivos que los conformaron. $\mathrm{Si}$ bien en algunos casos hubo reactivos que coincidieron en más de un factor, se revisó que

Tabla 1

Varianza de los factores

\begin{tabular}{|c|c|c|c|}
\hline \multirow[b]{2}{*}{ Factor } & \multicolumn{3}{|c|}{ Autovalores iniciales } \\
\hline & Total & Porcentaje de Varianza & Porcentaje Acumulado \\
\hline 1 & 9.48 & 28.95 & 28.95 \\
\hline 2 & 2.24 & 6.59 & 35.54 \\
\hline 3 & 1.71 & 5.05 & 40.60 \\
\hline 4 & 1.64 & 4.84 & 45.45 \\
\hline 5 & 1.38 & 4.06 & 49.51 \\
\hline 6 & 1.22 & 3.59 & 53.10 \\
\hline 7 & 1.13 & 3.33 & 56.44 \\
\hline 8 & 1.02 & 3.02 & 59.46 \\
\hline
\end{tabular}


todos los reactivos que lo conformaran tuvieran un sentido lógico. Después de realizar dicho análisis, de los factores iniciales, sólo cinco resultaron útiles debido a que los reactivos que los conformaron mostraron relación teórica; por último, éstos cinco factores fueron renombrados (tabla 2) y definidos de la siguiente manera:
1. Autoestima. El aprecio que se tiene uno mismo, el cual le permite aceptarse así mismo.

2. Moralidad. Llevar a cabo conductas que le permite tener una buena relación con las demás personas y le permitan una sana convivencia.

\section{Tabla 2}

\section{Estructura Factorial obtenida para la Escala de Resiliencia}

\begin{tabular}{|c|c|}
\hline $\begin{array}{l}\text { Factor 1. Autoestima } \\
\text { Alfa: } .85\end{array}$ & Pesos factoriales \\
\hline 1. Me he sentido a gusto conmigo mismo. & 0.75 \\
\hline 2. He llegado a quererme a mí mismo como soy. & 0.73 \\
\hline 4. He llegado a quererme a mí mismo. & 0.71 \\
\hline 3. Me he sentido orgulloso de mí mismo. & 0.65 \\
\hline 5. He sido alguien que confía plenamente en sí mismo. & 0.55 \\
\hline 10. He sido feliz. & 0.54 \\
\hline $\begin{array}{l}\text { Factor 2. Moralidad } \\
\text { Alfa: .77 }\end{array}$ & Pesos factoriales \\
\hline 14. Evito expresar críticas hacia los demás. & 0.79 \\
\hline 13. He tratado de no juzgar a las personas antes de conocerlas. & 0.76 \\
\hline 15. He intentado no envidiar lo que otros tienen o son. & 0.75 \\
\hline 16. He evitado imponer mis puntos de vista a los demás. & 0.52 \\
\hline $\begin{array}{l}\text { Factor } 3 \text {. Apoyo social } \\
\text { Alfa: } .60\end{array}$ & Pesos factoriales \\
\hline 29. He tenido alguien en mi familia que se preocupa por mí. & 0.64 \\
\hline 31. He contado con personas en quienes confío y me aceptan como soy. & 0.64 \\
\hline 25. He cultivado amistades verdaderas. & 0.62 \\
\hline $\begin{array}{l}\text { Factor } 4 . \text { Autopercepción } \\
\text { Alfa: . } 68\end{array}$ & Pesos factoriales \\
\hline 9. He reconocido que soy una persona que tiene un valor alto de sí mismo. & 0.65 \\
\hline 12. He observado que soy una persona inteligente. & 0.65 \\
\hline 11. Puedo describirme como alguien creativo. & 0.58 \\
\hline $\begin{array}{l}\text { Factor } 5 \text {. Independencia } \\
\text { Alfa: } .71\end{array}$ & Pesos factoriales \\
\hline 22. He decidido sobre mis acciones con libertad. & 0.73 \\
\hline 23. He sido independiente al tomar mis decisiones. & 0.71 \\
\hline 24. He podido realizar lo que he deseado. & 0.62 \\
\hline 21. He hecho lo que he querido. & 0.61 \\
\hline
\end{tabular}

Nota: Alfa de Cronbach global de la escala $=.87$. 
3. Apoyo social. Contar con personas en su entorno, las cuales le ayuden a sentirse apreciado y querido, debido a sus características personales.

4. Autopercepción. Percibirse como una persona con capacidades y habilidades que le permiten salir adelante en situaciones adversas.

5. Independencia. Sentirse capaz de poder actuar por cuenta propia, tomando por sí mismo las decisiones que considera adecuadas.

En la página anterior, se presentó la tabla con los reactivos que conformaron cada factor con su respectiva alfa de Cronbach.
Una vez obtenidas las propiedades psicométricas de los cambios efectuados, a la escala utilizada en este trabajo, se determinó el nivel de resiliencia de la muestra elegida.

Por último, se realizó una prueba t de student, para saber si existían diferencias entre hombres y mujeres, con respecto a cada uno de los factores obtenidos. Finalmente, el análisis reveló que no existen diferencias entre ambos (tabla 4).

Una vez descritos los resultados se pasará a compararlos con los obtenidos en el estudio de Villegas y Zamudio (2006), conforme con los objetivos del presente trabajo.

Tabla 3

Análisis descriptivos de los niveles de resiliencia de la muestra total

\begin{tabular}{lll}
\hline \multicolumn{1}{c}{ Factor } & \multicolumn{1}{c}{ Medias } & Desviación estándar \\
\hline Autoestima & 3.24 & .76 \\
Moralidad & 3.15 & 1.01 \\
Apoyo Social & 3.97 & .86 \\
Autopercepción & 3.28 & .84 \\
Independencia & 3.27 & .88 \\
Total & 3.53 & .65 \\
\hline
\end{tabular}

Tabla 4

Análisis de diferencias de los factores de resiliencia entre hombres y mujeres

\begin{tabular}{lccccc}
\hline & \multicolumn{5}{c}{ Medias } \\
\hline Factor & Mujeres & Hombres & t de student & gl & Sig. \\
\hline Autoestima & 3.28 & 3.21 & -0.74 & 248 & 0.11 \\
Moralidad & 3.21 & 3.09 & -0.93 & 248 & 0.34 \\
Apoyo social & 4.06 & 3.89 & -1.46 & 248 & 0.14 \\
Autopercepción & 3.35 & 3.23 & -1.12 & 248 & 0.26 \\
Independencia & 3.24 & 3.30 & 0.52 & 248 & 0.60 \\
Total & 3.357 & 3.5 & -.8 & 248 & 0.42 \\
\hline
\end{tabular}




\section{Discusión}

En relación al objetivo acerca de la modificación del formato de respuestas de la escala utilizada, ésta fue reportada en la sección de instrumentos. Conforme a los resultados obtenidos, ha mostrado confiabilidad y validez según las propiedades psicométricas pertinentes, a lo cual se añade un valor práctico, pues es más sencillo expresar rangos de respuesta en un formato tipo Likert de cinco rangos que en porcentajes con 10 rangos de respuesta.

En cuanto al nivel de resiliencia en la muestra de estudiantes de Oaxaca, este dio una media de 3.53, que con base en la escala de cinco rangos de respuesta convertida a porcentajes, da como resultados $70.6 \%$ de resiliencia, con mayor semejanza con el porcentaje de respuestas de la muestra de estudiantes Yucatecos (Villegas \& Zamudio, 2006), de $67.39 \%$. El resultado anterior permite establecer cierta consistencia de la escala utilizada, a pesar de los cambios en formato de respuestas y muestra estudiada.

En referencia al objetivo acerca de la estructura de respuestas en la muestra oaxaqueña, como se ha dicho antes, se encontraron los siguientes factores: Autoestima, Moralidad, Apoyo social, Autopercepción e Independencia, los cuales fueron semejantes los obtenidos en la muestra de estudiantes yucatecos: Autoestima, Moralidad, Apoyo social, Independencia, Relacionarse, Apoyo familiar y Optimismo. Estos datos además de evidenciar consistencia en la validación de la nueva escala, fortalecen el constructo de resiliencia, pues una cualidad común de ambas estructuras de respuestas, es la referencia que hacen las características personales de los individuos, como apoyo para salir a adelante en situaciones adversas.

El constructo de resiliencia ha recibido mucha atención en los últimos años, tal vez se deba a que alude a la capacidad de un sujeto para superar circunstancias de especial dificultad, gracias a sus cualidades mentales, de conducta y adaptación (Kreisler, 1996, citado en Manciaux, 2003). Recordemos que se le ha enmarcado dentro de la psicología positiva, cuyo fin es alcanzar una mejor calidad de vida y bienestar (Vera, 2006).

Con respecto a las diferencias entre hombres y mujeres, como se indicó anteriormente, en el presente trabajo fueron nulas, mientras que en la muestra de estudiantes yucatecos se encontraron diferencias en los factores de Autoestima e Independencia a favor de los hombres, lo cual es consistente con los resultados encontrados por González-Arratia, Valdez y Zavala (2008). De nuevo las semejanzas son mayores que las diferencias, aunque valdría la pena administrar la escala usada en el estudio de Villegas y Zamudio (2006), junto con la implementada en el presente trabajo, en una misma muestra para poder hacer comparaciones más precisas. Por lo pronto, la escala implementada en este estudio en su mayoría ha mostrado consistencia con la aplicada por Villegas y Zamudio (2006).

Finalmente, parafraseando a GonzálezArratia y Valdez (2012), los resultados encontrados muestran que la validación del 
nuevo formato del cuestionario implementado en una nueva muestra, cuenta con las propiedades psicométricas pertinentes y, por ende, es una medida válida del constructo de resiliencia.

\section{Referencias}

Ardila, R. (1971). Los pioneros de la psicología. Buenos Aires: Paidós.

Becerra-Martín, D., Espíndola-Mateos, J., \& Mézquita-Hoyos Y. (2012). Relación entre Resiliencia y Tenacidad. XX Congreso Mexicano de Psicología. Campeche, Campeche.

Carretero, B. R. (2010). Resiliencia. Una visión positiva para la prevención e intervención desde los servicios sociales. Nómadas. Revista Crítica de Ciencias Sociales y Jurídicas, 27. Recuperado de http://redalyc. org/articulo.oa?id=18113757004

Cassaretto, M., \& Martínez, P. (2009). Validación de la Escala del Sentido del Humor en estudiantes universitarios. Revista de Psicología, 27, 287-309.

Crespo, M., Fernández-Lansac, V., \& Soberón, C. (2014). Spanish Version of the CDRISC Resilience Scale for Chronic Stress Situations. Behavioral Psychology/ Psicologia Conductual, 22, 219-238. Recuperado de https://www.researchgate.net/ publication/260882710_Spanish_Version_ of_the_CD-RISC_Resilience_Scale_for_ Chronic_Stress_Situations

Friborg, O., Hjemdal, O., Rosenvinge, J. H., \& Martinussen, M. (2001). A new rating scale for adult resilience: What are the central protective resources behind healthy adjustment? International Journal of Methods in Psychiatric Research, 12(2), 6576.

González-Arratia, N., \& Valdez, J. (2012). Análisis factorial confirmatorio del cuestionario de resiliencia en una muestra de niños. En R. Díaz-Loving, S. Rivera \& I. Reyes (Eds.), La psicología social en México (Vol. XIV, pp. 676-681). México: Asociación Mexicana de Psicología Social.

González-Arratia, N., Valdez, J., \& GonzálezEscobar, S. (2014). Medición de la resiliencia. En M. Flores (Ed.), Aportaciones de la etnopsicología al estudio de la cultura y la personalidad (pp. 163-182). México: Universidad Autónoma de Yucatán.

González-Arratia, N., Valdez, J., \& Zavala, Y. (2008). Resiliencia en adolescentes mexicanos. Enseñanza e Investigación en Psicología, 13, 41-52. Recuperado de https://www.cneip.org/documentos/revista/ CNEIP_13_1/Gonzalez_Arratia_Lopez_ Fuentes.pdf

Grotberg, E. (2006). ¿Qué entendemos por resiliencia?, ¿cómo promoverla?, ¿cómo utilizarla? En E. Grotberg (Ed.), La resiliencia en el mundo de hoy. Cómo superar las adversidades (pp. 17-57). Barcelona: Gedisa. Hernández, R., Fernández, C., \& Baptista, P. (2015). Metodología de la investigación. México: McGraw-Hill. 
Hurtes, K. P., \& Allen, L. R. (2001). Measuring resiliency in Youth: The Resiliency Attitudes and Skills Profile. Therapeutic Recreation Journal, 35(4), 333-347.

Jew, C. L., Green, K. E., Kroger, J. (1999). Development and validation of a mesure of resiliency. Measurement and Evaluation in Counseling and Development, 32(2), 75-89.

Kalawski, J. P., \& Haz, A. M. (2003). Y... ¿dónde está la resiliencia? Una reflexión conceptual. Revista Interamericana de Psicología, 37, 365-372.

Manciaux, M. (2003). La resiliencia: resistir y rehacerse. España: Gedisa.

Matalinares, M., Arenas, C., Yaringaño, J., Sotelo, 1., Sotelo, N., Díaz, G., ... Tipacti, R. (2011). Factores personales de resiliencia y autoconcepto en estudiantes de primaria de Lima metropolitana. Revista de Investigación en Psicología, 14, 187-207. Recuperado de http://sisbib.unmsm.edu.pe/bvrevistas/ investigacion_psicologia/v14_n1/pdf/a12. pdf

Naranjo, R. (2010). Neurología de la resiliencia y desastres. Revista Cubana de Salud Pública, 36, 270-274. Recuperado de http:// scielo.sld.cu/scielo.php?script $=$ sci_arttext\& pid $=$ S0864-34662010000300013

Navarrete, J. (2009). Relación entre la resiliencia, los estilos de enfrentamiento y la depresión en estudiantes universitarios (tesis de maestría inédita). Universidad Autónoma de Yucatán, México.
Saavedra, G. E., \& Villalta, P. M. (2008). Medición de las características resilientes, un estudio comparativo en personas entre $15 \mathrm{y}$ 65 años. Liberabit. Revista de Psicología, 14, 31-40.

Seligman, M. (2003). La auténtica felicidad. España: Ediciones B.

Vera, B. (2006). Psicología positiva: una nueva forma de entender la psicología. Papeles del Psicólogo, 27, pp. 3-8.

Villegas, E. G., \& Zamudio, M. C. (2006). Resiliencia. Hacia la validación de una escala en adolescentes (tesis de licenciatura inédita). Universidad Autónoma de Yucatán, México.

Yates, A. (1982). Terapia del comportamiento. México: Trillas.

Recibido el 4 de mayo de 2015 Revisado el 8 de junio de 2015 Aceptado el 5 de agosto de 2016 